\title{
La Psicología como Ciencia y Profesión en el Afrontamiento del COVID-19
}

\author{
Psychology as a Science and Profession in coping with COVID-19
}

\author{
Alexis Lorenzo-Ruiz ${ }^{1 *}$, Kenny Díaz Arcaño ${ }^{2}$, \& Dionisio Zaldívar Pérez ${ }^{3}$ \\ 1 Profesor Titular de Psicología, Universidad de La Habana, Cuba. (iD https://orcid.org/0000-0003-4450-887X \\ 2 Facultad de Psicología, Universidad de La Habana, Cuba. (i) https://orcid.org/0000-0002-6019-1203 \\ 3 Profesor Titular y Emérito, Universidad de La Habana, Cuba. (DD https://orcid.org/0000-0002-0033-4815 \\ * Correspondencia: alexislorenzo64@gmail.com
}

Recibido: 22 abril 2020 | Aceptado: 7 junio 2020 | Publicado: 15 julio 2020

WWW.REVISTACARIBENADEPSICOLOGIA.COM

\section{Citar como:}

Lorenzo-Ruiz, A., Díaz, K., \& Zaldívar, D. (2020). La psicología como ciencia y profesión en el afrontamiento del COVID-19. Revista Caribeña de Psicología, 4(2), 153-165. https://doi.org/10.37226/rcp.v4i2.4815

\section{RESUMEN}

En diciembre del 2019, apareció un nuevo coronavirus en Wuhan, China, que rápidamente se extendió por todo el mundo. Fue nombrado SARS-CoV-2 y provoca el COVID-19. El presente artículo resulta de una revisión bibliográfica tipo narrativa sobre el tema. En los resultados se apreció la necesidad de comprensión psicológica y social en interrelaciones con otras dimensiones de la pandemia. Se identificaron manifestaciones clínicas psicológicas en los problemas relacionados con traumas y factores estresantes. Se abordó, además, la infodemia como reflejo de complejidad en la comunicación social, así como las interrelaciones entre tendencias, memoria histórica, niveles de entrenamiento y actuaciones profesionales propias de acompañamiento psicológico y social en desastres. Concluye resaltando aprendizajes y realidad de la psicología cubana como ciencia y profesión. Enfatiza su importancia en programas de salud, regularidad e interdisciplinariedad para la preparación de procesos permanentes en el ciclo de reducción de desastres, así como el rol protagónico de la comunidad y la voluntad política del Estado y el Gobierno de brindar un marco legal inclusivo de aspectos psicosociales en emergencias y desastres. Tales consideraciones teórico-metodológicas y práctico-instrumentales ya están siendo aplicadas en diversos programas y actividades en todo el país. Se recomienda la conformación del Observatorio Nacional de la Psicología Cubana en tiempos de COVID-19 y pautas para el trabajo en etapas futuras.

Palabras Claves: COVID-19; comportamiento humano; impacto psicológico; preparación

\begin{abstract}
In December 2019, a new coronavirus appeared in Wuhan, China, that quickly spread throughout the world. It was named SARS-CoV-2 and causes COVID-19. This article is a narrative-type bibliographic review on the subject. The results showed the need for psychological and social understanding in interrelationships with other dimensions of the pandemic. Clinical psychological manifestations were identified in problems related to trauma and stressors. The infodemic was also addressed as a reflection of the complexity in social communication, as well as the interrelationships between trends, historical memory, training levels and professional actions with a psychological and
\end{abstract}


social focus in disasters. It concludes by highlighting learning and reality of Cuban Psychology as a science and profession. It emphasizes its importance in health, regularity and interdisciplinarity programs for the preparation of permanent processes throughout the cycle of disaster reduction, as well as the leading role of the community and the political will of the State and Government in order to provide an inclusive legal framework of psychosocial aspects in emergencies and disasters. Such theoretical-methodological and practical-instrumental considerations are already being applied in various programs and activities throughout the country. The development of the National Observatory of Cuban Psychology in the time of COVID-19 and guidelines for work in future stages are recommended.

Keywords: COVID-19; behavior human; psychological impact; preparation

\section{INTRODUCCIÓN}

La propagación de epidemias ha estado presente a lo largo de la historia de la humanidad. Algunos ejemplos a citar van desde la peste que devastó Europa en el siglo XIV y los brotes mortales de cólera en el siglo XIX (Infosalud, 2020; Infobae, 2020), hasta la epidemia de SIDA de los años 80 (Cardona-Arias \& Higuita-Gutiérrez, 2014); el virus del Ébola (Bitanihirwe, 2016) que se extendió desde África, la desastrosa propagación del Zika en Brasil y el MERS-CoV (AlRabiaah et al., 2020). Históricamente, estos brotes han causado retos e impactos inconmensurables en el orden de lo psicológico, socioeconómico y político.

En diciembre del 2019 en la República Popular China, surgió una nueva enfermedad infecciosa causada por el Síndrome Respiratorio Agudo Coronavirus 2 (SARS-CoV-2, por sus siglas en inglés). Los primeros casos de contagiados con el nuevo Coronavirus, se reportaron en la ciudad de Wuhan, provincia de Hubei. El SARS-CoV-2, dio lugar a la enfermedad "COVID-19". Esta nueva enfermedad, ha ocasionado un impacto mundial sin precedentes, tanto por lo imprevisto y la rapidez de su propagación a nivel internacional, como por el elevado número de fallecidos. La población mundial está asustada, y con frecuencia se acerca a comportamientos paranoides. Las consecuencias socioeconómicas son casi impredecibles y no muy esperanzadoras global y regionalmente (Infobae 2020; Inchausti et al., 2020; BBC, 2020).

El COVID-19 como fenómeno amenazante, ratifica la necesidad de nuevas valoraciones a nivel mundial de las enfermedades no transmisibles y transmisibles. En la actualidad, se refuerza la idea de la amenaza, singularidad y excepcionalidad que representa este virus. A nivel de la subjetividad humana, esta temática está muy ligada a la memoria histórica de nuestra civilización. La imperiosa necesidad de este material consiste en que las personas de todo el mundo estén informadas adecuadamente, tanto en los síntomas, como en su propia comprensión del fenómeno en sí: "Pandemia COVID-19". Expectativa conformada como regularidad humana cuando se vivencian situaciones de epidemias.

Esta situación es catalogada, acorde a la Directiva No. 1 del Consejo de Defensa Nacional (CDN) de Cuba, como un desastre de origen sanitario. Durante la apreciación del peligro de epidemias en un territorio, debe prestarse especial atención al estado higiénico sanitario de las ciudades $y$, fundamentalmente, analizar los factores vulnerables de las mismas, que inciden en el surgimiento y propagación de enfermedades. Ante una emergencia de tal magnitud, parte de la población responde con medidas "curativas y/o de solución inmediata" como tratamiento; este es el comportamiento de la mayoría de las personas ante una amenaza "real" a la salud humana. Al no existir aún un remedio farmacológico eficaz, en este trabajo abordaremos algunas consideraciones psicológicas de este fenómeno. En aras de ofrecer, en abreviada manera, un consenso en pos de ayudar a mejorar, y quizá a resolver algunas de las inquietudes más comunes, el presente artículo se integra y es reflejo de la respuesta de la ciencia cubana ante el COVID-19 (Canal Caribe, 2020).

Este es un estudio de revisión bibliográfica tipo narrativa (en momentos posteriores, se presentará una versión más avanzada y en profundidad, con acceso a bases de datos especializadas). Se analizan, de forma combinada, publicaciones anteriores en el área, y recientes investigaciones producto del quehacer profesional con poblaciones ya damnificadas por esta epidemia. 


\section{NECESIDAD DE LA COMPRENSIÓN PSI- COLÓGICA Y SOCIAL}

Si interrelacionamos las complejidades y matices de cada una de estas cuestiones, se impone la necesidad de incorporar los modelos y criterios de las ciencias sociales y humanísticas (Vommaro, 2020), desde cuyos resultados será posible obtener explicaciones y sólidos argumentos sobre las dimensiones sociales, políticas y económicas de la pandemia. La pandemia COVID-19 ha producido una fuerte tensión internacional multinivel, en especial la relacionada a las políticas y recursos en salud (existentes o no), ante la contingencia del evento. Este nuevo fenómeno constituye en sí un grave problema global con consecuencias no solo para salud, sino también en lo económicofinanciero, geopolítico y en tensiones derivadas, de todos los tipos, a nivel local e internacional (Murueta, 2020; Santini, 2020).

En este campo del conocimiento, ha predominado comprender el comportamiento humano en situaciones críticas, extremas, emergencias y desastres, desde una tendencia con énfasis hacia la posible o preponderante enfermedad psíquica psicopatológica (Barlow \& Durand, 2015). Ello incluye al Tercer Glosario Cubano de Psiquiatría (GC-3) y la Guía Latinoamericana de Diagnóstico Psiquiátrico (Asociación Psiquiátrica de América Latina, 2012). Ya en el año 1989, se pudo leer un análisis epidemiológico y social de 2000 estudios importantes realizado por Seaman y Leivesley (1989). En el mismo, fueron descritas alrededor de 200 posibles reacciones psicológicas ante un desastre. Estos datos ya fueron corroborados posteriormente por otras investigaciones y estudios en estos últimos años (Benyakar, 2003; World Health Organization, 2005).

En el caso de el COVID-19 resulta llamativo estudiar estas variables dado su ritmo acelerado de propagación y la creciente magnitud de impactos, incluyendo las de índole psicosocial. Tal aseveración, nos lleva a valorar su carácter de contingencia, variable presente entre la emisión de una determinada conducta y la llegada de las consecuencias. Este fenómeno explica si un determinado comportamiento se adquiere, se mantiene, se elimina, o incluso si es más resistente a la extinción. Muchos de los problemas graves en salud, o de difícil de intervención-prevención, por ejemplo, adicciones o enfermedades de transmisión sexual, son posibles de entender por la contingencia. En pocas palabras, cuando los sujetos percibimos que entre la emisión de una determinada conducta y la eventual llegada de las consecuencias (sean positivas o negativas) es poco probable, más difícil, o pasa mucho tiempo para que este comportamiento se adquiera o modifique (Urzúa et al., 2020). Tales criterios se reflejan desde la tendencia de una baja percepción de riesgo hasta el mito de la invulnerabilidad en muchas poblaciones, quienes aún hoy no concientizan ni lo que está aconteciendo, ni mucho menos a dónde podría parar este grave problema de salud mundial.

Al ser tan compleja y variable esta situación a nivel mundial, es posible integrar el conjunto de estas manifestaciones clínicas psicológicas alrededor de los posibles trastornos relacionados con traumas y factores de estrés en su fase aguda. Los síntomas van desde inquietud, angustia, nerviosismo, temores, incertidumbre y ansiedad, pudiendo llegar a cuadros de ansiedad generalizada, crisis de pánico, tristeza, apatía, depresión y desesperanza. Ello incluye manifestaciones psicofisiológicas tales como dolores de diferente naturaleza, insomnio, pesadillas, disminución o pérdida del apetito, abandono de hábitos higiénicos y cambios bruscos en la sexualidad, entre otros. Igualmente, se observan problemas de carácter conativo en el sistema de relaciones sociales, proyectos de vida, motivación, autoestima, autovaloración, estilos de afrontamiento, percepción de riesgo, representaciones sociales, estereotipos y creencias (racionales e irracionales), así como en el rendimiento y/o estabilidad de sus principales actividades personales, estudiantiles, creativas, productivas y otras variables de vital importancia. Estas cuestiones ya han sido reflejadas en anteriores investigaciones especializadas en este campo (Domínguez et al., 2012; González, 2012; Ventura, 2012), llegándose a hacer profundos análisis desde una comprensión personológica (Reis et al., 2016; 2016a).

Tales preceptos reafirman cuánto urge profundizar y conocer el tema de la comprensión social del COVID-19 (Shah, 2020) y su valoración, siguiendo los preceptos psicológicos del comportamiento humano en situaciones de emergencias y desastres (Figueroa \& Marín, 2016; Muldoon et al., 2019; World Health Organization, 2019). En el área de las epidemias también existen sólidos estudios sobre el tema (Gardner 
\& Moallef, 2015; Taylor, 2019), que han permitido que en los últimos años se perciban notables cambios en esta concepción. Han ido transitando a una comprensión más holística desde lo psicosocial (Amaro et al., 2017; Castro et al., 2014; Figueroa \& Marín, 2016; Ruiz, 2015), hasta lograrse la necesaria inclusión de la temática en las prácticas psicológicas basadas en la evidencia científica (Lorenzo, 2000). Esta multiplicidad de criterios, en mayor o menor medida afines, es posible integrarlos metodológica y conceptualmente desde los contenidos de la "Ecuación Modelo del Psiquismo Cuidado/Auto-Cuidado" (ver Figura 1).

Esta ecuación puede ser subdividida simbólica y esquemáticamente en 5 elementos:

1. Los eventos: cada uno de ellos tiene sus particularidades en momentos de la vida habitual, y como es lógico, cambian durante y posteriormente a las situaciones críticas, como acontece hoy con la pandemia COVID-19. Unos son esperados, nos avisan con tiempo suficiente; mientras otros resultan abruptos, muy rápidos e inesperados. Por sus particularidades generan mayores o menores tipos de pérdidas. No obstante, por muy novedoso y complejo que hoy resulte encuadrar aquí el Cuidado/Auto Cuidado, constituye un reto encontrar sus regularidades y especificidades en comparación con anteriores etapas que hayamos experimentado individual o colectivamente en el transcurso de nuestras vidas.
2. Las personas: van a pensar, actuar y reflexionar ser generalmente (en mayor o menor medida), de un modo u otro en lo cotidiano y en las crisis, condicionándose directa e indirectamente, según sus individualidades-(edad, sexo, nivel escolar, sistema de creencias, identidad cultural, experiencias / vivencias previas, preparación, etc.), las cuales, de alguna manera, potenciarán el posible impacto, consecuencias y repercusiones ulteriores.

3. El entorno: como ser biopsicosocial, cada persona es producto de diversos factores y aspectos que están directa e indirectamente interrelacionados. La familia, la comunidad y la escuela son de los más relevantes a considerar.

4. Las posibles respuestas: se constituyen como patrones por sus regularidades en cada una de las etapas del ciclo vital y el proceso complejo de formación de la personalidad.

5. Los Programas / Protocolos: si nos quedamos en este análisis hasta los anteriores elementos, en el comportamiento humano, ante cada nueva crisis, prevalecería el enfoque ensayo-error, reaccionar cuando algo ya ocurrió. Sin embargo, las lecciones aprendidas y optimizadoras, aun con la complejidad y variabilidad de las situaciones (tal y como acontece en época de COVID-19) se convierten en manuales de buenas prácticas. Cuba y

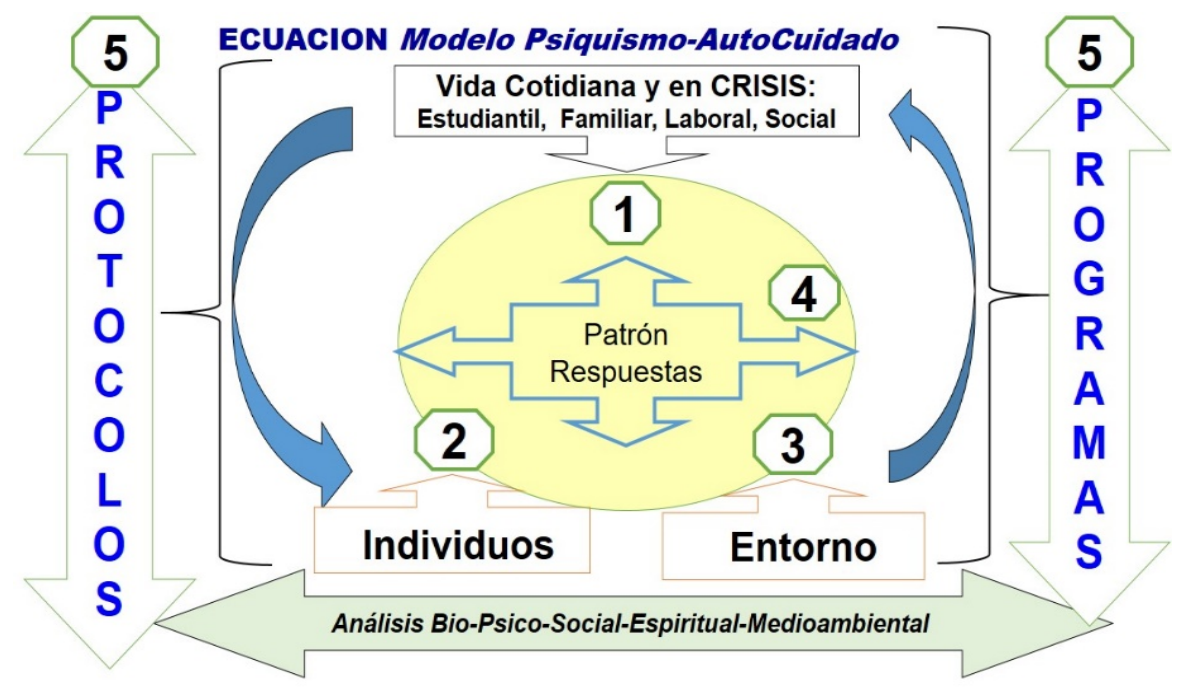

Figura 1. Ecuación Modelo del Psiquismo con énfasis en el Cuidado/Auto-Cuidado. 
otras regiones, los han recibido por parte de autoridades y profesionales de China y aquellos países donde se han ido venciendo para generar las que son imprescindibles. Cada uno de ellos constituye un producto armónico del quehacer multi-transdisciplinar. Están basados en evidencias profesionales científicas que combinan de forma creativa el saber con el quehacer popular. De ese modo, resultan integrados y sistematizados en importantes y necesarios Programas/Protocolos. Estos, a su vez, se convierten en brújulas orientadoras y luces iluminadoras del camino de cada persona e institución para combatir las situaciones y exigencias que vivimos a nivel mundial. Tal y como se ha dicho, ya se vienen aplicando exitosa y efectivamente en muchos escenarios laborales de Cuba y del mundo (Lorenzo, 2003; 2006).

Tal comprensión destaca que los desastres constituyen un hecho inesperado y de por sí catastrófico, provocando grandes pérdidas humanas y materiales. Los individuos y sus comunidades, en mayor o menor medida relacionados con el desastre, lo perciben como un evento vital estresante, una especie de situación amenazante y de conflicto, la cual conlleva a una ruptura y modificación del ciclo vital de los damnificados y del equilibrio hombre-entorno en una sociedad concreta (Lorenzo, 2009; 2011).

El comportamiento humano ante los desastres, no resulta en la mayoría de los casos, como consecuencia de una respuesta automática, ni de un conjunto de manifestaciones psicotraumáticas, porque el proceso de estresogénesis no funciona como una relación tan fatalista de tipo lineal "causa-efecto" en los desastres. Por lo tanto, el ser humano, mostrará un comportamiento "normal" ante las exigencias críticas, amenazantes, conflictivas y desbordantes denominadas "anormales" en situaciones de desastres, por su esencia. Esto es así, dadas las posibilidades incalculables de resiliencia para reestructurar y movilizar los mecanismos necesarios de adaptación y readaptación humana (Brooks et al., 2020).

En reafirmación de ello, la Organización Mundial de la Salud (OMS) alertó que el impacto psicológico (a corto, mediano y largo plazo) del COVID-19 en la sociedad, no debe ser ignorado (Cabrera, 2020;
Inchausti et al., 2020). El coronavirus llega, entonces, como un "multishock" a una humanidad con múltiples enfermedades psicológicas, de mayor o menor severidad, dependiendo de las circunstancias particulares de vida de cada persona y de cada colectividad (Murueta, 2020).

En la literatura por épocas y lugares del mundo a través del tiempo, son descritos innumerables casos de brotes emocionales, asociados a comportamientos diversos. Estos han ocurrido en contextos caracterizados por determinados conflictos o amenazas, y de manera particular en el seno de grupos en los cuales, por alguna razón, ha existido una gran tensión o estrés. Esto se ha expresado a partir de la respuesta de uno de sus miembros y posteriormente se ha extendido a los otros integrantes del colectivo, o de la comunidad, como un intento de salida o respuesta a la situación. Este fenómeno de réplica emocional y de respuestas observadas en otras personas -donde se encuentran presente la imitación y la empatía-, se explica a nivel del Sistema Nervioso Central (SNC).-Esto es, debido a la existencia de las llamadas "Neuronas Espejos", que cumplen una función de reflejo y amplificación de los estímulos exteriores al individuo y posibilitan la aparición de estas respuestas empáticas o imitativas (por simpatía) (Rodríguez et al., 2011).

Tales evidencias han mostrado cómo el estado emocional de un sujeto puede verse afectado por la exposición al sufrimiento, las quejas y otras expresiones emocionales de sus contactos sociales, lo que significa que el estado de ánimo y el estrés pueden propagarse de persona a persona, por un proceso llamado "contagio emocional" (Bartholomew \& Zaldivar, 2018; Inchausti et al., 2020).

En este sentido, un elemento importante a resaltar es la llamada "infodemia". Esta se ha desarrollado alrededor de la pandemia del coronavirus y tiene sus fuentes en mensajes tóxicos que se transmiten a través de las redes sociales y a la saturación de información relacionada con su impacto y consecuencias, que reflejan situaciones críticas como las presentadas en determinados lugares como Guayaquil, New York, etc. Esto provoca que se incrementen los temores, ansiedades y sentimientos de amenaza e indefensión, que alimentan en los individuos vulnerables el estado de 
tensión y estrés, afectando el bienestar psicológico de los mismos.

La Organización Mundial de la Salud (OMS) definió la infodemia como el peligro de la desinformación durante la pandemia, o la sobreabundancia informativa de noticias falsas, maliciosas y a su rápida propagación entre las personas y medios. De modo que el exceso de información incrementa el estrés, la angustia y los temores infundados, además de la incertidumbre, la impotencia y la confusión. Ello complica el descanso y hace disminuir las defensas inmunológicas, tan necesarias para afrontar un proceso viral (Santini, 2020; BBC, 2020; Infocop, 2020; Infocop, 2020a).

Otra problemática de gran resonancia corresponde a la "cuarentena obligatoria" (tanto a nivel de la comunidad científica, como de la población en general) con su especificidad a nivel psicosocial, lo cual es analizado también desde diferentes áreas del conocimiento. El comportamiento humano en estas condiciones de pandemia se asocia a la imperiosa necesidad de mantener y respetar el distanciamiento; con el pedido explícito de las autoridades sanitarias de mantenerse todo el tiempo en sus propias casas y reducir al máximo posible las actividades sociales. Esta recomendación es de carácter sanitario, basada en los criterios epidemiológicos, los cuales están asociados al margen de un tiempo que no exceda el periodo máximo de incubación de la enfermedad.

Por tales criterios, resulta esperado que una cuarentena produzca efectos psicológicos negativos, como el estrés agudo, la confusión, el aumento de la agresión y la violencia, entre otros. También pueden emerger la rememoración de eventos adversos históricos, la oposición a las medidas sanitarias, incrementarse el narcisismo y la omnipotencia, las sociopatías y otras, que pueden requerir límites singulares, su elaboración, contención o límites externos. Además, su prolongación indefinida, puede incrementar el temor a la enfermedad viral, el aburrimiento, el estrés por las pérdidas ocasionadas, la caída o complicación de proyectos, entre otros. Prevalecen manifestaciones emocionales tales como: miedo, frustración, enojo, ambivalencia, desorganización, aburrimiento, tristeza, sentimiento de soledad, sensación de encierro y ansiedad. También destacan, cómo la sobreinformación, amplifica las emociones en cuarentena y crea efectos adversos, pues la gente tiende a consumir información que coincide con su pensamiento previo, por ende, si son negativos, van a consumir noticias pesimistas (Gaeta \& Lorenzo, 2012; Rodríguez et al., 2010).

Se observan latentes este tipo de inquietudes en el área de las comunicaciones y sus matices psicosociales. En tiempos de pandemia, exigen continuar investigándose por su magnitud, diversidad y demostrados impactos. Para ello, tener en cuenta resultados de investigaciones cubanas (Lorenzo, 2009a; Lorenzo \& Guerrero, 2017; González, 2012) y latinoamericanas (León, 2012; Murueta, 2020). Tales criterios resaltan un tema vital por atender y dar seguimiento en tiempos de el COVID-19. Se trata de los aspectos éticos y de la corresponsabilidad profesional en el desempeño de cualquiera de estas acciones desde la psicología, otras ciencias o disciplinas, y del voluntariado en situaciones de desastres (Vommaro, 2020; Inchausti et al., 2020).

Por el carácter globalizado del momento que vivimos, y en particular de las comunicaciones, la red de redes, hace un llamado a no potenciar ni multiplicar el efecto Dunning-Kruger (Kruger \& Dunning, 1999). Este puede resumirse en una frase: "cuanto menos sabemos, más creemos saber". Es un sesgo cognitivo según el cual las personas con menos habilidades, capacidades y conocimientos tienden a sobrestimar esas mismas habilidades, capacidades y conocimientos. Como resultado, estas personas suelen convertirse en ultracrepidianos, gente que opina sobre todo lo que escucha sin tener idea, pero pensando que sabe mucho más que los demás (Kruger \& Dunning, 1999).

Estas cuestiones, las cuales podríamos encuadrar como de carácter no deseado o generalmente negativo, reafirma la alta incidencia del fenómeno conocido como "Segundo Desastre". Enunciado por Erikson en 1976 como el impacto de la desorganización social en periodo ulterior al desastre (Cohen \& Ahearn, 1980, p. 33). Todo el accionar de programas $\mathrm{u}$ otras actividades en estas circunstancias deben estar basados en los preceptos de la ética y la bioética para situaciones de emergencias y desastres (León, 2012). Cada una de estas cuestiones está reflejada en las primeras publicaciones que abordan los resultados preliminares en China y otros países (Brooks et al., 2020; Cabrera, 2020; Duan \& Zhu, 2020; IASC, 
2020; Legido-Quigley et al., 2020; Puthanveettil, 2020; Wang et al., 2020).

En estudios revisados en personas infectadas con anteriores epidemias producidas por coronavirus (e.g., Severe Acute Respiratory Syndrome, SARS; y Middle East Respiratory Syndrome, MERS), fueron identificados 3 grupos de riesgo siguiendo la morbilidad psicológica durante y posterior a el COVID-19: 1) Personal profesional de la salud; 2) Individuos, quienes como resultado de estas crisis, ya han sido expuestos a eventos traumáticos, tales como: afectaciones o pérdidas entre sus seres queridos, amenazas a su salud $u$ otras áreas vitales de sus vidas como estudio y trabajo; 3) Personas con preexistencia de diferentes cuadros psicopatológicos, en especial aquellos considerados como trastornos psiquiátricos severos (Brooks et al., 2020).

\section{NECESIDAD DE PROGRAMAS DE ACOM- PAÑAMIENTO PSICOLÓGICO Y SOCIAL}

Tales consideraciones, concernientes a la pandemia de el COVID-19 y sus matices psicosociales, reafirman la imperiosa necesidad de organizar diferentes modos tanto de comprensión como de acompañamiento relativos al impacto psicológico en tales circunstancias (IASC, 2007; Puthanveettil, 2020; World Health Organization, 2005; 2019).

Para entender la urgencia y vigencia de la psicología aplicada a problemas de la salud humana, cabe destacar que ha sido esencialmente el resultado natural de: (a) las controversias con el modelo biomédico tradicional y sus limitaciones en la práctica actual en salud, (b) el fracaso de los sistemas sanitarios por una concepción restrictiva y descontextualizadora de la salud; y, (c) la posibilidad de comprender cómo variables sociales y psicológicas (creencias, conductas, emociones, actitudes, apoyo social, etc.) mediatizan los problemas de salud como factores desencadenantes, facilitadores o moduladores del proceso saludenfermedad (Grau et al., 2012).

Por lo tanto, constituye una urgencia tener en cuenta todo este material bibliográfico descrito. El cual, al ser de tan variado formato teórico, metodológico, práctico e instrumental, recomendamos para su mejor comprensión y posterior aplicación, trabajarlo siempre en tres posibles dimensiones de trabajo y sus posibles fuentes:
1. Marcos conceptuales y criterios metodológicos internacionales. Contenidos de los Manuales y/o Guías basadas en investigaciones y documentaciones de Organismos Internacionales, principalmente del sistema de las Naciones Unidas (World Health Organization, 2005; 2019). Importantes aportes aquí se reconocen por parte de la Federación Internacional de la Cruz Roja y Media Luna Roja (2003).

2. Evidencias científicas y experiencias profesionales desde la psicología en situaciones de desastres. Publicaciones de los Colegios, Asociaciones e instituciones de la Psicología a nivel internacional (APA, 2013; Everly, 2020; Figueroa \& Marín, 2016).

3. Lecciones aprendidas autóctonas: resultados de trabajos producidos en Cuba (Amaro et al., 2017; Castro et al., 2014; González, 2012; Pell del Rio et al., 2017; Rolo et al., 2014; Sánchez et al., 2012; Sandoval et al., 2010; Ventura, 2012; Díaz \& Lorenzo, 2016). Este criterio, es hoy posible aplicarlo en la mayoría de los países al contar con motivantes y consistentes experiencias nacionales en esta área.

Este razonamiento reafirma los hallazgos descritos por Duan y Zhu (2020), quienes reconocen en sus resultados la imperiosa necesidad de programas de intervención psicológica especializados en las diferentes fases de la pandemia de COVID-19. Estos autores resumen sus aprendizajes en los siguientes aspectos:

1. Déficits del sistema de salud en términos de recursos humanos y materiales.

2. Subestimación social (a corto y largo plazo) de las consecuencias psicológicas de la pandemia, y de los recursos limitados para trabajar con ellos.

3. Pobre planificación y coordinación de las intervenciones psicológicas, especialmente cuando se aplican a varios niveles por diferentes profesionales.

4. Finalmente, también hay un riesgo asociado a las respuestas tempranas a las crisis. Este conlleva a la proliferación de intervenciones y marcos relacionados con la saturación de asistencias psicológicas, bien intencionadas, pero no basadas en evidencias, comúnmente por ONGs, o un tercer sector (Duan \& Zhu, 2020). 
Tales criterios, igualmente, demuestran validez y consistencia en los resultados, que son satisfactorios acorde a las investigaciones anteriormente descritas; $\mathrm{y}$ al funcionamiento de los programas ya aplicados en poblaciones damnificadas por el COVID-19 a nivel mundial desde enero a abril 2020. Por lo tanto, al integrarse la experiencia cubana con énfasis en las lecciones aprendidas internacionales, se considera imposible poder afrontar la pandemia COVID-19, sin contar con pautas concretas sobre las diversas modalidades propias del acompañamiento psicológico y social en desastres (Ver Figura 2).

En el caso de Cuba, la respuesta y accionar desde su comunidad científica no se ha hecho esperar (Arés, 2020; Castellanos, 2020; Lorenzo, 2020). Este accionar se diseña, implementa, valida y desarrolla de forma coordinada y supervisada por las Sociedades Científicas Cubanas de Psiquiatría y Psicología de conjunto (Armas, 2020).

En este momento, a nivel mundial, se observa un cambio rotundo en el necesario y ético desempeño profesional de la psicología en situaciones de pandemia. Se requiere concientizar y aplicar diversos cambios en los paradigmas teórico-metodológicos, mayor comprensión del comportamiento humano en situaciones críticas desde la psicopatología, las posibles comorbilidades de enfermedades y problemas de salud (incluyendo a la salud mental) en los sistemas de encuadres de las prácticas clínicas-psicoterapéuticas y otras modalidades especializadas de atención a estas poblaciones.
Se refiere, en estas circunstancias, al tránsito inmediato del modelo "caso a caso", a la actuación profesional a través de terceros como una demanda y necesidad sentida en tiempos de COVID 19. Es decir, se trata de modos de actuación profesional en la psicología más de forma y modos indirectos que directos y personalmente, cara a cara. Son descritos como modos de actuación desde la Telepsicología, como todo un conjunto de actividades de acompañamiento psicológico y de otras modalidades a través de la red y/o redes online (Lluch-Canut, 2020; Urzúa et al., 2020).

Por lo tanto, van apareciendo nuevos y complejos retos ante la comunidad científica de la psicología, la psiquiatría, trabajo social, y otras áreas de las ciencias de la salud y sociales-humanísticas. Todas ellas con estrechos vínculos para diseñar respuestas con inmediatez, eficiencia, eficacia, pertinencia, y sobre todo sostenibilidad en el ámbito clínico y de la salud desde un abordaje al máximo social. En consonancia con estos criterios, urge continuar el desarrollo de programas locales de atención primaria de salud y el perfeccionamiento de los recursos biomédicos, los cuales no serán plenamente efectivos por sí solos sin una nueva forma de pensamiento en salud que considere los factores psicosociales (Lorenzo, 2009b). Para la psicología, el desafío es recuperar toda la evidencia científica disponible, que por décadas han generado sus actores, para incorporarla en forma clara y precisa y así contribuir en los problemas y desastres en salud en este caso (Urzúa et. al., 2020).

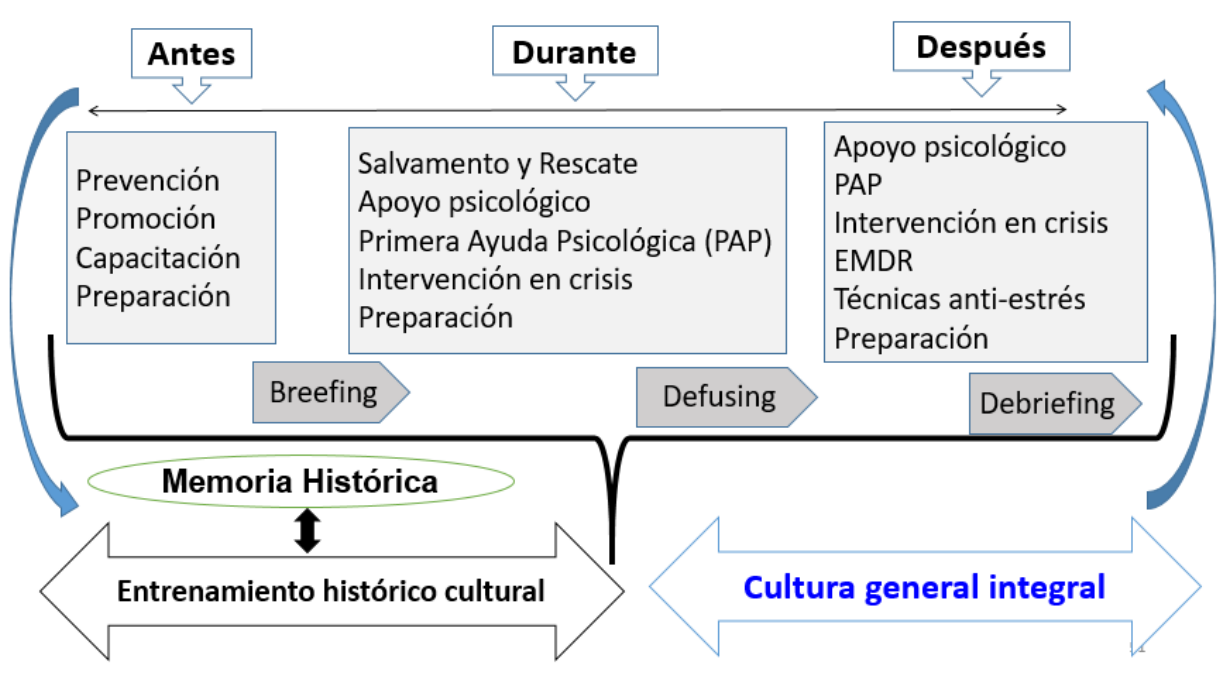

Figura 2: Modalidades acompañamiento psicológico y social en desastres 
Ese desafío, ratifica la aplicabilidad de los aprendizajes de Cuba desde la psicología como ciencia y profesión en programas de salud (Grau et al., 2012; Lorenzo et al., 2012; Sánchez et al., 2014), siguiendo, entre otros, al menos cuatro criterios como tendencias: (a) la multi y transdisciplinariedad (Betancourt, 2013; Rodríguez et al., 2011); (b) la preparación con carácter de proceso permanente en todo el ciclo de reducción de desastres (Lorenzo, 2009); (c) rol protagónico de la comunidad (Amaro et a., 2017); (d) la voluntad política del Estado y Gobierno con un marco legal inclusivo de los aspectos psicosociales en emergencias y desastres (Lorenzo et al., 2012). Esto marca la sustancial diferencia de la evolución de la pandemia en Cuba, en comparación con la realidad desfavorable en otros entornos, incluyendo a países con muchos más recursos y niveles de desarrollo. Estas experiencias y consideraciones en conjunto permiten reafirmar las novedades científicas relativas al Ciclo del Proceso de Reducción de los Desastres y sus nexos con la ciencia psicológica (Ver Figura 3).

En la Figura 3, hay un llamado de atención a las lecciones aprendidas en este sentido, particularmente a la necesidad de seguir investigando sobre cada momento y etapas propias de la ocurrencia de estos fenómenos. Se escuchan / leen en los medios de difusión masiva algunas de estas preguntas permanente y repetidamente: ¿Cuándo termina?, ¿Cuánto tiempo va a durar? ¿Y después de el COVID-19 cómo va a ser la vida? ¿Cuándo volveremos a la normalidad y la cotidianeidad?, etc. Se trata de un conjunto de procesos mucho más complejos de lo que pudiese parecer, al cual denominamos "Readaptación humana en el camino de retorno paulatino a la normalidad y cotidianeidad".

Todas estas consideraciones teórico-metodológicas y práctico-instrumentales, entre otros aspectos psicológicos y sociales, constituyen una reafirmación y apego a las pautas descritas en las orientaciones sanitarias emitidas por el Gobierno y Estado Cubano a través del Protocolo de Actuación Nacional del Ministerio de Salud Pública (MINSAP). Estas reafirman las pautas de los protocolos internacionales y los enriquecen con las experiencias cubanas en este tipo de situaciones epidemiológicas.

A modo de recomendaciones para futuras acciones de trabajo e investigaciones del tema, resaltamos las siguientes:

- Profundizar en las particularidades psicológicas y sociales de la comprensión de la pandemia COVID-19 en la población cubana.

- Profundizar en el proceso salud-enfermedad (y de la salud mental también) en todas sus dimensiones en personas diagnosticadas con COVID-19 y todos los que de un modo u otro participan en este complejo ámbito. A su vez, delimitando la caracterización del cuadro subjetivo interno de la enfermedad en personas diagnosticadas.

\section{GIGLO DEL PROGESO DE REDUGGIÓN DE DESASTRES}

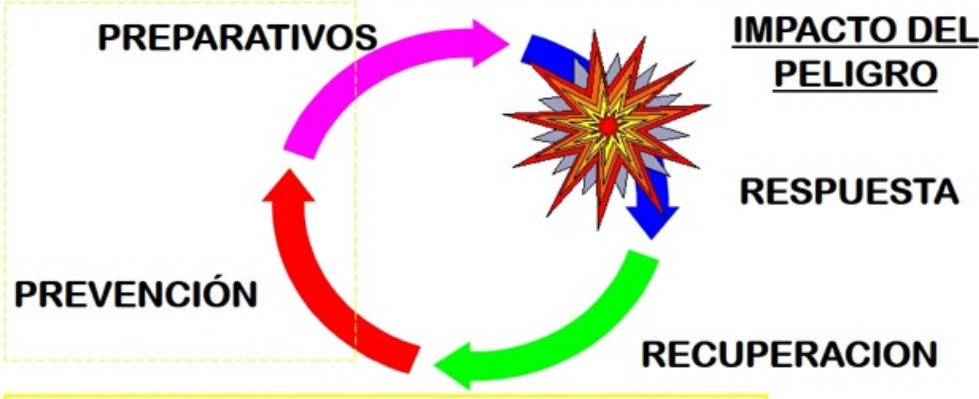

(Rehabilitación + Reconstrucción = Recuperación)

Delimitar Proceso específico “RE-Adaptación”

Figura 3: Ciclo del Proceso de Reducción de los Desastres y sus nexos con la Psicología. 
- Continuar investigando sobre las particularidades de los impactos psicológicos (a corto, mediano y largo plazo) de los cambios en el comportamiento humano asociados a la COVID 19; incluyendo al distanciamiento, la cuarentena y al aislamiento que se le pide a la población como medida de protección sanitaria.

- Delimitar aspectos en común y diferencias sustanciales del comportamiento humano en tiempos de COVID-19, en relación a otras situaciones de emergencias y desastres.

- Sistematizar las diferentes modalidades de prácticas de actuación psicológica en este ámbito, delimitando las particularidades de lo que acontece internacionalmente y lo autóctono en cada país.

- De modo particular, continuar trabajando en la protocolización de modos de actuación desde la psicología para la atención a los equipos que cumplimentan la atención a la salud de personas con el COVID-19.

- Continuar trabajando en la conformación del Observatorio Nacional de la Psicología Cubana como reflejo de su accionar como ciencia y profesión en tiempos de COVID-19, para, en etapas futuras, socializar, divulgar y valorar sus resultados.

\section{CONCLUSIONES}

La psicología como ciencia en tiempos de el COVID-19, resulta un binomio imprescindible para favorecer la salud y el bienestar humano. Por esto se trabaja intensamente en cada país desde la psicología, para identificar y profundizar sobre aspectos medulares del comportamiento humano en tiempos de

\section{REFERENCIAS}

Al-Rabiaah, A., Temsah, M.H., Al-Eyadhy, A.A., Hasan, G.M., AlZamil, F., AlSubaie, S., \& Somily, A.M. (2020). Middle East Respiratory Syndrome-Corona Virus (MERS-CoV) associated stress among medical students at a university teaching hospital in Saudi Arabia. Journal of Infection and Public Health, 13(5), 687691. https://doi.org/10.1016/j.jiph.2020.01.005

Amaro, A. J., Barroso, C.T., \& Lorenzo, A. (2017). Instrumentos y metodologías de los diagnósticos de la vulnerabilidad psicosocial de la comunidad y de la vulnerabilidad familiar, ante situaciones de desastres meteorológicos. Revista Electrónica Cuadernos de Crisis. www.cuadernosdecrisis.com/docs/2017/numero16vol1_2017_3_instrumentos.pdf pandemia. Hay ya sólidos hallazgos obtenidos en las investigaciones descritas -mucho más divulgadasque han aplicado cuestiones propias desde los métodos epidemiológico, sociológico y clínico. Sin embargo, en este trabajo se llama la atención y reafirma la urgencia de complementar la comprensión y actuación profesional también desde los preceptos de la ciencia psicológica. Pero no desde alguna de sus áreas en detrimento de otras, ni de forma absoluta. Se trata aquí de incorporar al método psicológico en su dimensión integradora y sistematizada, enfatizándose en la multi y transdisciplinariedad. Ninguna de sus áreas y experiencias resultan en menor o mayor significado necesarias, todas son bienvenidas y con posibilidades de inmediata aplicabilidad.

En ese sentido, la Psicología en Emergencias y Desastres -como una especialización ya reconocidaamerita ser más incorporada a este tipo de trabajo por poseer suficiente evidencia científica y validadas metodologías de desempeño profesional por más de 30 años de existencia. Sin esta evidencia, no cabe la menor duda, que se podría llegar tarde y/u ofrecer un abordaje insuficiente o distorsionado para continuar trabajando en el afrontamiento ante el COVID-19 a nivel mundial. Finalmente, es necesario tener en cuenta todos los aspectos mencionados aquí para diseñar nuevas líneas de trabajo e investigación en el presente y futuro; legitimando aún más el papel de la psicología en tiempos de pandemia y ajustándolo a las características y cultura de cada país o región en cuestión.

Financiamiento: La presente investigación no fue financiada por alguna entidad ni patrocinador.

Conflicto de Intereses: No existe conflicto de intereses de parte de los autores de la investigación.

American Psychiatric Association, DSM-5 Task Force. (2013). Diagnostic and statistical manual of mental disorders: DSM-5 $5^{\mathrm{TM}}$ (5th ed.). American Psychiatric Publishing, Inc. https://doi.org/10.1176/appi.books.9780890425596

Arés, P. (2020). Luz larga para la esperanza y luz corta para la ansiedad. Cubadebate. http://www.cubadebate.cu/noticias/2020/04/02/luz-larga-para-la-esperanza-y-luz-corta-parala-ansiedad/\#.XqTEdGD_rIU

Armas, I. (2020). Ante el COVID-19 psiquiatras y psicólogos de Cuba ratifican su compromiso con el bienestar y la salud humana. Agencia Cubana de Noticias. 
Asociación Psiquiátrica de América Latina. (2012). Guía Latinoamericano para el Diagnóstico Psiquiátrico. Versión Revisada (GLADP-VR). Asociación Psiquiátrica de América Latina.

Barlow, D., \& Durand, V.M. (2015). Abnormal psychology an integrative approach (5th Ed.). Cengage.

Bartholomew, R., \& Zaldivar, D. (2018). Chasng ghost in Cuba; Is Mass psychogenic illness masquerading as an acoustical attack? International Journal of Social Psychiatry, 64(5), 413-416. https://doi.org/10.1177\%2F0020764018766185

BBC News. (2020). Coronavirus: cómo el miedo a la enfermedad COVID-19 está cambiando nuestra psicología. Consultado el 17 Abril 2020. https://www.bbc.com/mundo/noticias-52191660

Benyakar, M. (2003). Lo Disruptivo. Amenazas individuales y colectivas: el psiquismo ante guerras, terrorismos y catástrofes sociales. Biblos.

Betancourt, J.A. (2013). Modelo transdisciplinario para la investigación en salud pública. Revista Panamericana de Salud Pública, 34(5), 2013, 359-363.

Bitanihirwe, B.K.(2016). Monitoring and managing mental health in the wake of Ebola. Commentary. Annali dell'Istituto Superiore Di Sanita, 52(3),320-322. https://doi.org/10.4415/ANN_16_03_02

Brooks, S.K., Webster, R.K., Smith, L.E., Woodland, L., Wessely, S., Greenberg, N., \& Rubin, G.J. (2020). The psychological impact of quarantine and how to reduce it: rapid review of the evidence. The Lancet, 395(10227), 912-920. https://doi.org/10.1016/S0140-6736(20)30460-8

Cabrera, J.E. (2020). La Organización Mundial de la Salud (OMS) alerta que el impacto psicológico del COVID-19 en la sociedad no debe ser ignorado. Sección "Lo que debes saber hoy". https://www.biobiochile.cl/especial/lo-que-debes-saberhoy/2020/03/27/oms-alerta-que-el-impacto-sicologico-del-COVID-19-en-la-sociedad-no-debe-ser-ignorado.shtmlr

Canal Caribe. (2020). Directivos e investigadores de Cuba informan sobre esfuerzo científico contra el COVID-19. Disponible en http://www.canalcaribe.icrt.cu/directivos-e-investigadoresde-cuba-informan-sobre-esfuerzo-cientifico-contra-la-COVID19/

Cardona-Arias, J. A., \& Higuita-Gutiérrez, L. F. (2014). Impact of HIV/AIDS on quality of life: Meta-Analysis 2002-2012. Revista Especializada Salud Pública, 88(1), 87-101. https://doi.org/10.4321/s1135-57272014000100006

Castellanos, R. (2020). Ante el Coronavirus prevenir sin excesos de ansiedad. https://www.sicologiasinp.com/psicologia-clinica/anteel-coronavirus-prevenir-sin-excesos-de-ansiedad/

Castro, M., Melluish, S., \& Lorenzo, A. (2014). Cuban Internationalism-An alternative form of globalisation. Journal International Review of Psychiatry, 26(5), 595-601. https://doi.org/10.3109/09540261.2014.920770

Cohen, R.E., Ahearn, F.L. (1980). Manual de la atención de salud mental para víctimas de desastres. C.V.

Díaz, K., \& Lorenzo, A. (2016). Conocimientos e impacto psicológico de los desastres en estudiantes de psicología: resultados de una investigación. Revista Integración Académica en Psicología, 4(12), 45-56.

Domínguez, B.; Pennebaker, J.W.; \& Olvera López, Y. (2008). Estrés Postraumático (EPT). Editorial Trillas.

Duan, L., \& Zhu, G. (2020). Psychological interventions for people affected by the COVID-19 epidemic. Lancet Psychiatry, 7(4), 300-302. https://doi.org/10.1016/S2215-0366(20)30073-0

Everly, G.S. (2020). Psychological First Aid (PFA) To Expand Mental Health Support and Foster Resiliency in Underserved and Access-Compromised Areas. Crisis, Stress, and Human Resilience, 1(4), 227-232.

Federación Internacional de Sociedades de la Cruz Roja, Media Luna Roja. (2003). Apoyo Psicológico basado en la Comunidad. Manual de Formación. http://www.cruzroja.org/documentos/docs/manual_apoyo_psicologico_sp.pdf

Figueroa, R., \& Marín, H. (2016). Desafíos que enfrenta Latinoamérica en salud mental en emergencias y desastres. http://www.sochped.cl/noticias/prioridades-para-la-proteccion-de-la-salud-mental-despues-de-desastres-en-latinoamerica-y-el-caribe

Gaeta, N., \& Lorenzo, A. (2012). Salud y Desastres. Experiencia Cubana $V$. La incursión de la comunicación en la prevención de desastres: el caso cubano. ECIMED. http://www.bvs.sld.cu/libros/salud_desastre_v/cap_08.pdf

Gardner, P.J., \& Moallef, P. (2015). Psychological impact on SARS survivors: Critical review of the English language literature. Canadian Psychology/Psychologie canadienne, 56(1), 123-135. https://doi.org/10.1037/a0037973

González, R. (2012). La Psicoterapia con víctimas de desastres. Ediciones Dampsa. Habana, Cuba.

Grau, J.A., Infante, O.E., \& Díaz, J.A. (2012). Psicología de la salud cubana: Apuntes históricos y proyecciones en los inicios del siglo XXI. Revista Pensamiento Psicológico ,10(2), 83-10.

Inchausti, F., Hasson-Ohayon, I., Macbeth, A., \& Dimaggio, G. (2020). Psychological Intervention and COVID 19 What we Know so far and What We can do. Journal of Contemporary Psychotherapy, May 27, 1-8. https://doi.org/10.1007/s10879-02009460-w

Infobae. (2020). De la peste negra al coronavirus: cuáles fueron las pandemias más letales de la historia. https://www.infobae.com/america/mundo/2020/03/18/de-la-peste-negra-al-coronaviruscuales-fueron-las-pandemias-mas-letales-de-la-historia/

Infocop. (2020). El impacto psicológico de la cuarentena y cómo reducirlo, según un estudio. http://www.infocop.es/view_article.asp?id=8630

Infocop. (2020a). Recomendaciones de la Asociación Americana de Psicología (APA) para manejar la ansiedad que suscitan las noticias sobre el Coronavirus. http://www.infocop.es/view_article.asp?id=8595

Kruger, J., \& Dunning, D. (1999). Unskilled and unaware of it: How difficulties in recognizing one's own incompetence lead to inflated self-assessments. Journal of Personality and Social Psychology, 77(6), 1121-1134. https://doi.org/10.1037//0022-3514.77.6.1121 
Legido-Quigley, H., Mateos-García, J.T., Campos, V.R., GeaSánchez, M., Muntaner, C., \& McKee, M. (2020). The resilience of the Spanish health system against the COVID-19 pandemic. Lancet Public Health, 5(1), 251-252. https://doi.org/10.1016/S2468-2667(20)30060-8

León, F.J. (2012). Bioethics in catastrophe situations such as earthquakes. Revista Médica de Chile, 140(1), 108-112.

Lluch-Canut, M.T. (2020) (Coord.). Decálogos de Salud Mental Positiva. Adaptados a diferentes situaciones y/o personas afectadas por circunstancias de la pandemia de Coronavirus COVID-19. Barcelona: Colección OMADO, Deposito Digital de la Universidad de Barcelona. http://hdl.handle.net/2445/155018

Lorenzo A. (2003). Apoyo psicológico en desastres. En, Monografía Gerencia de Desastres en Cuba Una compilación de Artículos. http://wwwcardin.uwimona.edu.jm:1104

Lorenzo, A. (2000). Psychological Base International Rehabilitation Programs for People who had suffered after The Biggest Critical Incident [Thesis]. National University of Internal Affairs, Kharkov.

Lorenzo, A. (2005). Rol de la psicoterapia integrada individual/grupal a un programa de rehabilitación para niños damnificados por desastres. Revista Electrónica de Psicología Iztacala, 8(3), 5871.

Lorenzo, A. (2006). La Psicología en el Programa cubano de atención a personas afectadas por el accidente de Chernobil. En, Monografía Hacer y Pensar la Psicología, Compilación y Prólogo Ana María del Rosario Asebey y Manuel Calviño. Editorial Caminos, La Habana-Cuba, pp. 129-157.

Lorenzo, A. (2006). Reflexiones sobre la evolución del quehacer psicológico en el tema de emergencias y desastres. Análisis de la experiencia en Cuba. Revista Cuadernos de Crisis, 5(2), 7-37.

Lorenzo, A. (2009). Teoría y prácticas en actividades de preparación psicosocial para emergencias y desastres en Cuba y América Latina. Memorias del III Congreso Nacional de Innovaciones en Psicología y Salud Mental. Escuela de Psicología de la Universidad de Tunja.

Lorenzo, A. (2009a). Especificidades de la comunicación en condiciones de incidentes críticos de gran envergadura. Psicología de la comunicación en el siglo XXI: 10 años de desarrollo. Ciencias Sociales, 24-32.

Lorenzo, A. (2009b). Lecciones aprendidas en la organización de programas de ayuda e intervención psicológica para las situaciones de emergencias y desastres en Cuba. Salud y Desastres. Experiencia Cubana I. ECIMED.

Lorenzo, A. (2011). Aspectos psicológicos y sociales de la labor de enfermería en emergencias y desastres. Monografía Psicología y Práctica Profesional de Enfermería.

Lorenzo, A. (2020). Una visión desde la Psicología en Cuba a el COVID19. Presa Latina. https://www.prensa-latina.cu/index.php?o=rn\&id=356720\&SEO=una-vision-desde-la-psicologia-en-cuba-a-la-pandemia-COVID-19-info-y-video

Lorenzo, A., \& Guerrero, E.A. (2017). Afectaciones psicológicas en personal de primera respuesta: ¿Trastorno por Estrés Postraumático o Estrés? Revista Puertorriqueña de Psicología, 28(2), 330343.
Lorenzo, A., Gómez, C., Sánchez, Y.Y., \& Balseiro, J. (2012). Primeras experiencias del Programa de Mitigación del daño psicosocial en la población infanto- juvenil afectada por el terremoto en Haití. Cuba: Salud y Desastres. Experiencia Cubana V. ECIMED.

Lorenzo, A., Gómez, C., Ventura, R.E., Mesa, G. (2012). Lineamientos para la salud mental en emergencias y desastres en Cuba. Bases metodológicas para la elaboración e implementación de la Indicación No. 20 del Ministro de Salud Pública de Cuba. Salud y Desastres. Experiencia Cubana V. ECIMED. http://www.bvs.sld.cu/libros/salud_desastre_v/cap_01.pdf

Muldoon, O.T., Haslam, S.A., Haslam, C., Cruwys, T., Kearns, M., \& Jetten, J. (2019). The social psychology of responses to trauma: social identity pathways associated with divergent traumatic responses. European Review of Social Psychology, 30(1), 311-348. https://doi.org/10.1080/10463283.2020.1711628

Murueta, M. E. (2020). Salud psicológica en tiempos del Coronavirus 19. Comunicación personal (Notas para la conferencia impartida por Zoom los días 7 y 8 de abril de 2020).

Pell del Río, S.M, Lorenzo-Ruiz, A., \& Torres, A. (2017). Determinación de la percepción de riesgo de la población ante los productos químicos peligrosos. Revista Cubana de Salud Pública, 43(2), 139-148.

Puthanveettil, N. (2020). The Guidelines AISC. Aid workers put rights first in mind care. http://www.tsunamiresponsewatch.org/trw/2007/03/13/aid-workers-put-rights-firstin-mind-care/

Reis, A. M., Carvalho, L. F., \& Elhai, J. (2016). Predictive capacity of pathological personality traits to the Post-traumatic Stress Disorder. Psicologia em Pesquisa, 10(2), 85-92. http://dx.doi.org/10.24879/201600100020063

Reis, A. M., Carvalho, L. F., \& Elhai, J. (2016a). Relationship between PTSD and pathological personality traits in context of disasters. Psychiatry Research, 241, 91-97. https://doi.org/10.1016/j.psychres.2016.04.099

Rodríguez, P. M., Del Pino, D. A., \& Alvaredo, R. B. (2011). De lo psicológico a lo fisiológico en la relación entre emociones y salud. Revista Psicología Científica, 13(13). http://www.psicologiacientifica.com/relacion-emociones-y-salud

Rodríguez, Y., Lorenzo, A., Mesa, G., \& Salabarría, C. (2010). Salud y Desastres. Experiencia Cubana III. Desarrollo de capacidades locales en situaciones de desastres en la Isla de la Juventud, una experiencia para compartir. ECIMED, 137-143. http://www.bvs.sld.cu/libros/salud_desastre_tomo03/indice_p.htm

Rolo, A.I., Lorenzo, A., Pérez, C., Quiñonez, R.E., \& Rolo, F.M. (2014). Percepción de Riesgo de Desastres de origen natural en Estudiantes de Medicina. Salud y Desastres. Experiencia Cubana VIII. ECIMED. http://bvscuba.sld.cu/libros-de-autores-cubanos/listado-de-titulos-en-orden-alfabetico/\#S\%20\%20Uhttp://www.bvs.sld.cu/libros/salud_desastre_viii/

Ruiz, E.M. (2015). El triage psicológico: ¿Una herramienta para el psicólogo de emergencias? Revista Electrónica Cuadernos de Crisis, 14(1). http://www.cuadernosdecrisis.com/docs/2015/numero14vol1_2015_triage_psicologico.pdf 
Sánchez, Y., Lorenzo, A., Ventura, R., Martínez, C., \& Balseiro, J. (2014). Estrategia de implementación de los Lineamientos sobre salud mental en situaciones de desastres en Cuba. Salud y Desastres. Experiencia Cubana VIII. ECIMED, 69-76. http://bvscuba.sld.cu/libros-de-autores-cubanos/listado-de-titulos-en-orden-alfabetico/\#S\%20-\%20Uhttp://www.bvs.sld.cu/libros/salud_desastre_viii/

Sánchez, Y.Y., Lorenzo, A., Gómez, C., Balseiro, J., \& Ventura, R.E. (2012). El Equipo de Salud Mental en la Brigada Médica de Primera Respuesta para situaciones de desastres. Salud y Desastres. Experiencia Cubana V. ECIMED. http://www.bvs.sld.cu/libros/salud_desastre_v/cap_04.pdf

Sandoval, S., Lorenzo, A., \& Sandoval, J.E. (2010). Conociendo a los niños ante los desastres. Salud y Desastres. Experiencia Cubana III. ECIMED, 364-369. http://www.bvs.sld.cu/libros/salud_desastre_tomo03/indice_p.htm

Santini, O.A. (2020). Coronavirus y medidas psicosanitarias. Facultad Psicología de la Universidad de Córdoba. Comunicación Personal.

Seaman, J., \& Leivesley, C. (1989). Epidemiología de los Desastres Naturales. C.V.

Shah, H. (2020). Global problems need social science. Nature, 577, 295. https://doi.org/10.1038/d41586-020-00064-x

Taylor, S. (2019). The psychology of pandemics. Preparing for the next global outbreak of infectious disease. Cambridge Scholars Publishing.
Tercer Glosario Cubano de Psiquiatría. (2001). Diagnóstico y Clasificación de los Trastornos Mentales. Adaptación para Cuba de la $10 \mathrm{ma}$. (GC-3). Revisión de la Clasificación Internacional de Enfermedades Mentales (CIE-10). Editorial Ciencias Médicas.

Urzúa, A., Vera-Villarroel, P., Caqueo-Urízar, A., \& Polanco-Carrasco, R. (2020). La Psicología en la prevención y manejo del COVID-19: Aportes desde la evidencia inicial. Revista Terapia Psicológica, 38(1), 103-118. https://teps.cl/index.php/teps/article/view/273/323

Ventura, R. (2012). Trastornos psicosociales en desastres. Temas para entrenamiento. Editorial Ciencias Médicas.

Vommaro, P. (2020). Las dimensiones sociales, políticas y económicas de la pandemia. Pensar la Pandemia. Observatorio social del Coronavirus CLACSO. www.clacso.org

Wang, C., Pan, R., Wan, X., Tan, Y., Xu, L., Ho, C. S., \& Ho, R. C. (2020). Immediate psychological responses and associated factors during the initial stage of the 2019 Coronavirus Disease (COVID-19) Epidemic among the general population in China. International Journal of Environmental Research and Public Health, 17(5), 1729. https://doi.org/10.3390/ijerph17051729

World Health Organization. (2005). World Health Organization Assessment Instrument for Mental Health Systems. Emergencies (WHO-AIMS-E 1.0).

World Health Organization. (2019). WHO mental health Gape Action Plan Programmed (mhGAP). www.who.int

\section{Obra bajo licencia de Creative Commons Atribución 4.0 Internacional (CC BY 4.0).} (C) 2020 Autores. 